\title{
Condensation and interaction range in harmonic boson traps: A variational approach
}

\author{
J. Tempere, ${ }^{1}$ F. Brosens, ${ }^{1}$ L. F. Lemmens, ${ }^{2}$ and J. T. Devreese ${ }^{1,2}$ \\ ${ }^{1}$ Departement Natuurkunde, Universiteit Antwerpen UIA, Universiteitsplein 1, B2610 Antwerpen, Belgium \\ ${ }^{2}$ Departement Natuurkunde, Universiteit Antwerpen RUCA, Groenenborgerlaan 171, B2020 Antwerpen, Belgium
}

(Received 17 May 1999; published 9 March 2000)

\begin{abstract}
For a gas of $N$ bosons interacting through a two-body Morse potential a variational bound of the free energy of a confined system is obtained. The calculation method is based on the Feynman-Kac functional projected on the symmetric representation. Within the harmonic approximation a variational estimate of the effect of the interaction range on the existence of many-particle bound states, and on the $N-T$ phase diagram is obtained.
\end{abstract}

PACS number(s): 03.75.Fi, 05.30.Jp, 32.80.Pj

\section{INTRODUCTION}

In this paper, we continue the investigation proposed in [1] and started in [2], on the influence of realistic interactions on the expression for the free energy of the interacting Bose system. The method we propose is distinct from other treatments in two main aspects. First, it allows for the treatment of finite-range interatomic potentials whereas nearly all theoretical studies relying on a mean-field description have focused on a two-body contact potential. Second, the exact quantum statistics, both of the condensate and of the noncondensate atoms, is treated analytically at arbitrary temperature.

The experimental realization of Bose-Einstein condensation (BEC) in systems of trapped, interacting bosonic atoms [3-5] has led to renewed theoretical efforts $[1,2,6-14]$ to understand the properties of Bose gases. The need to go beyond the contact potential (also discussed in [15-17]) appears because the ground-state energy of a contact potential with negative scattering length (relevant for the ${ }^{7} \mathrm{Li}$ ) is not bounded from below. The present study of finite-range potentials, based on a variational principle resulting from the Jensen-Feynman inequality, avoids this artifact. This variational principle (originally formulated by Feynman [18] to treat the problem of an electron in a polarizable medium) is extended in Sec. II to treat many-body systems with finiterange interactions, thereby incorporating the quantum statistics of the particles analytically. To investigate interaction potentials different from the contact potential, the $T$-matrix formulation used in $[7,8,16]$ can also be applied. In this formulation, the limit of the contact potential is given by the long-wavelength limit. In a sense, the present method is the "real space" complement of the "momentum space" $T$-matrix calculation: the knowledge of the pair correlation function $g(\mathbf{r})$ of the model system in the present approach allows for effective study of the effects of the spatial dependence of the interaction potential.

The system that we analyze in the present paper consists of a fixed number of bosonic atoms in a parabolic confinement. The interatomic interaction studied in detail in this paper is a Morse potential where the parameters are determined by the scattering length and the neutral atomic radius. Although in the most recent experiments [19] mixtures of gases with different spin states are examined, we consider only the spin-polarized Bose gas in the present analysis. The results of the path integral variational method elaborated in Sec. II, applied to the spin-polarized gas of bosons interacting through a Morse potential, are reported in Sec. III, and the specific case of lithium is investigated using the experimentally derived interatomic potential (from spectroscopy measurements of Abraham et al. [20-22]). The discussion of the results and a comparison with other methods are presented in Sec. IV.

\section{STUDY OF THE MORSE POTENTIAL}

The essential property of realistic interatomic interactions is that atoms repel at short distances and attract when they are some distance apart. In this section, we study the effect of the Morse potential $v_{2}(\mathbf{r})$, which has these two main characteristics, on a collection of bosonic atoms

$$
\frac{v_{2}(\mathbf{r})}{U}=\left(1-e^{-\left(r-r_{0}\right) / L}\right)^{2}-1,
$$

where the vector $\mathbf{r}$ (with length $r$ ) is the difference in position vectors of the two atoms, and $r_{0}$ is a parameter that determines the range of the potential. $U$ is a parameter that determines the strength of the potential, and $L$ is a parameter that determines the "stiffness" of the potential near its minimum. These parameters are related to experimentally observable quantities. For the Morse potential the number of bound levels $N_{l e v}$ and the scattering length $a_{\text {scat }}$ are given by [23]

$$
\begin{aligned}
& N_{\text {lev }}=\left[\frac{1}{2}(1-\sqrt{8 U} L)\right], \\
& a_{\text {scat }}=U L^{3} \frac{e^{r_{0} / L}-16}{4 e^{-r_{0} / L}},
\end{aligned}
$$

where the square brackets denote the largest positive integer smaller than the expression between the brackets. The remaining parameters of Eq. (1) are determined by a leastsquares method using the shape of an experimentally determined potential [20-22].

\section{A. The Feynman-Kac variational method}

The Feynman-Kac functional is defined as an average over a Brownian motion $\{R(t) ; t \geqslant 0\}$ with a variance that is 
proportional to that of standard Brownian motion by a factor $\sqrt{\hbar / m}$, see, e.g., [24]. The Brownian motion provides the sample paths in a $3 N$-dimensional configuration space (whose elements $\left\{\mathbf{r}_{1}, \mathbf{r}_{2}, \ldots, \mathbf{r}_{N}\right\}$ are denoted by $r$ ). The initial and final points of these paths are incorporated in the averaging symbol $E_{r}$ by the index and by an indicator function $I\left(R(t)-r^{\prime}\right)$. Using these concepts, a propagator written as

$$
K\left(r, t ; r^{\prime}\right)=E_{r}\left[I\left(R(t)-r^{\prime}\right) \exp \left\{-\frac{1}{\hbar} \int_{0}^{t} V(R(s)) d s\right\}\right]
$$

satisfies the Bloch equation for distinguishable particles

$$
\frac{\partial}{\partial t} K\left(r, t ; r^{\prime}\right)=\frac{\hbar}{2 m} \nabla^{2} K\left(r, t ; r^{\prime}\right)-\frac{1}{\hbar} V(r) K\left(r, t ; r^{\prime}\right),
$$

with

$$
\lim K\left(r, t ; r^{\prime}\right)=\delta\left(r-r^{\prime}\right)
$$

Using the projection techniques borrowed from [25] and applied to confined systems in $[1,26]$, it is easy to see that the partition function for $N$ identical particles at an inverse temperature $\beta=1 /\left(k_{B} T\right)$ is given by

$$
\begin{aligned}
Z(N, \beta)= & \frac{1}{N !} \int d r E_{r}\left[\sum_{P} \xi^{P} I(R(\beta)-P(r))\right. \\
& \left.\times \exp \left\{-\frac{1}{\hbar} \int_{0}^{\beta} V(R(s)) d s\right\}\right],
\end{aligned}
$$

where $P$ denotes permutations of the particle coordinates. A summation over all elements of the permutation group is taken. Every permutation contributes a factor $\xi^{P}$, which is -1 for odd permutations of fermions and 1 in all other cases. If the partition function $Z_{0}(N, \beta)$ and some static correlation functions of a model system, with potential energy $V_{0}$, can be calculated analytically,

$$
\begin{aligned}
Z_{0}(N, \beta)= & \frac{1}{N !} \int d r E_{r}\left[\sum_{P} \xi^{P} I(R(\beta)-P(r))\right. \\
& \left.\times \exp \left\{-\frac{1}{\hbar} \int_{0}^{\beta} V_{0}(R(s)) d s\right\}\right],
\end{aligned}
$$

then this knowledge can be used to derive an upper bound for the free energy $F=-\ln [Z(N, \beta)] / \beta$, relying on the JensenFeynman inequality

$$
\begin{aligned}
Z(N, \beta)= & \frac{1}{N !} \int d r E_{r}\left[\sum_{P} \xi^{P} I(R(\beta)-P[r])\right. \\
& \times \exp \left\{-\frac{1}{\hbar} \int_{0}^{\beta} V_{0}(R(s)) d s\right\} \\
& \left.\times \exp \left\{-\frac{1}{\hbar} \int_{0}^{\beta}\left[V(R(s))-V_{0}(R(s))\right] d s\right\}\right] \\
= & Z_{0}(N, \beta)\left\langle\operatorname { e x p } \left[-\frac{1}{\hbar} \int_{0}^{\beta}[V(R(s))\right.\right. \\
& \left.\left.\left.-V_{0}(R(s))\right] d s\right]\right\rangle \\
\geqslant & Z_{0}(N, \beta) \exp \left[-\frac{1}{\hbar} \int_{0}^{\beta}\left\langle V(R(s))-V_{0}(R(s))\right\rangle d s\right] .
\end{aligned}
$$

In this expression, the angular brackets denote the quantum statistical expectation value

$$
\begin{aligned}
\langle A(R(\tau))\rangle= & \frac{1}{Z_{0}(N, \beta)} \int d r E_{r}\left[\sum_{P} \xi^{P} I(R(\beta)-P[r])\right. \\
& \left.\times \exp \left\{-\frac{1}{\hbar} \int_{0}^{\beta} V_{0}(R(s)) d s\right\} A(R(\tau))\right] .
\end{aligned}
$$

We consider a spin polarized-gas of bosons interacting through a two-body potential $v_{2}$ such as Eq. (1) and confined by an anisotropic parabolic potential. The potential energy of this system is given by

$$
V=\frac{m}{2} \sum_{j=1}^{N}\left[\Omega_{x}^{2} x_{j}^{2}+\Omega_{y}^{2} y_{j}^{2}+\Omega_{z}^{2} z_{j}^{2}\right]+\sum_{j=1}^{N} \sum_{l=j+1}^{N} v_{2}\left(\mathbf{r}_{j}-\mathbf{r}_{l}\right),
$$

with $m$ the mass of the particles, and $\mathbf{r}_{j}=\left\{x_{j}, y_{j}, z_{j}\right\}$ the position of the $j$ th boson. The partition function, the density, and the pair correlation function for a model system with potential energy $V_{0}$ given by

$$
V_{0}=\sum_{j=1}^{N} \frac{m}{2}\left[\Omega_{x}^{\prime 2} x_{j}^{2}+\Omega_{y}^{\prime 2} y_{j}^{2}+\Omega_{z}^{\prime 2} z_{j}^{2}\right]+\sum_{j=1}^{N} \sum_{l=j+1}^{N} \frac{\kappa}{2}\left(\mathbf{r}_{j}-\mathbf{r}_{l}\right)^{2}
$$

were derived analytically in Refs. $[1,26]$ for the isotropic case. Substituting the real potential energy for the spinpolarized gas of interacting bosons (11), and the potential energy of the trial system (12) in the inequality (9), one finds 


$$
\begin{aligned}
F \leqslant & F_{0}+\sum_{j=1}^{N} \frac{m}{2}\left[\left(\Omega_{x}^{2}-w_{x}^{2}\right)\left\langle x_{j}^{2}\right\rangle+\left(\Omega_{y}^{2}-w_{y}^{2}\right)\left\langle y_{j}^{2}\right\rangle\right. \\
& \left.+\left(\Omega_{z}^{2}-w_{z}^{2}\right)\left\langle z_{j}^{2}\right\rangle\right]-\frac{N \kappa}{2} N\left\langle\mathbf{R}^{2}\right\rangle \\
& +\frac{1}{2}\left\langle\sum_{j=1}^{N} \sum_{l \neq j=1}^{N} v_{2}\left(\mathbf{r}_{j}-\mathbf{r}_{l}\right)\right\rangle,
\end{aligned}
$$

where $F_{0}=-\ln \left[Z_{0}(N, \beta)\right] / \beta$ is the free energy of the model system with partition function $Z_{0}(N, \beta), w_{x, y, z}^{2}=\left(\Omega_{x, y, z}^{\prime}\right)^{2}$ $+N \kappa / m$, and $\mathbf{R}=(1 / N) \sum_{j=1}^{N} \mathbf{r}_{j}$ is the center-of-mass coordinate. Using the pair correlation function

$$
g(\mathbf{r})=\frac{1}{N(N-1)} \int \frac{d^{3} \mathbf{k}}{(2 \pi)^{3}} e^{-i \mathbf{k r}}\left\langle\sum_{j=1}^{N} \sum_{l \neq j=1}^{N} e^{i \mathbf{k}\left(\mathbf{r}_{j}-\mathbf{r}_{l}\right)}\right\rangle,
$$

the expectation value of the two-body potential $v_{2}$ can be rewritten

$$
\left\langle\sum_{j=1}^{N} \sum_{l=j+1}^{N} v_{2}\left(\mathbf{r}_{j}-\mathbf{r}_{l}\right)\right\rangle=\frac{N(N-1)}{2} \int d \mathbf{r} v_{2}(\mathbf{r}) g(\mathbf{r}),
$$

and hence the variational free energy can be written as

$$
\begin{aligned}
F \leqslant & F_{0}+\frac{m}{2} \sum_{j=1}^{N}\left[\left(\Omega_{x}^{2}-w_{x}^{2}\right)\left\langle x_{j}^{2}\right\rangle+\left(\Omega_{y}^{2}-w_{y}^{2}\right)\left\langle y_{j}^{2}\right\rangle\right. \\
& \left.+\left(\Omega_{z}^{2}-w_{z}^{2}\right)\left\langle z_{j}^{2}\right\rangle\right]-\frac{N \kappa}{2} N\left\langle\mathbf{R}^{2}\right\rangle \\
& +\frac{N(N-1)}{2} \int d \mathbf{r} v_{2}(\mathbf{r}) g(\mathbf{r}) .
\end{aligned}
$$

This is the central variational formula that we will use to find the thermodynamical properties of the spin-polarized, parabolically confined, interacting bosons. The essential role of the pair correlation function in the evaluation of the expectation value of the interaction potential is clear from Eq. (16).

The required building blocks for the variational free energy (the expectation values and the pair correlation function for the bosonic case), obtained previously $[1,26]$ for the isotropic case, have to be extended to the case of an anisotropic confinement potential. This anisotropic generalization is documented in the Appendix. The resulting expression for the variational free energy is

$$
\begin{aligned}
F \leqslant & -\frac{1}{\beta} \ln \left(Z_{0}(N, \beta)\right)+\sum_{i=x, y, z}\left\{\frac{1}{\beta} \ln \left(\frac{\sinh \left(\beta \hbar \Omega_{i}^{\prime} / 2\right)}{\sinh \left(\beta \hbar w_{i} / 2\right)}\right)+\frac{\hbar\left[\Omega_{i}^{2}-\left(\Omega_{i}^{\prime}\right)^{2}\right] \operatorname{coth}\left(\beta \hbar \Omega_{i}^{\prime} / 2\right)}{4 \Omega_{i}^{\prime}}\right\} \\
& +\sum_{i=x, y, z} \frac{\hbar\left[\Omega_{i}^{2}-w_{i}^{2}\right]}{4 w_{i}}\left[\left(\sum_{l=1}^{N} \frac{Z_{0}(N-l, \beta) \operatorname{coth}\left(\beta \hbar w_{i} l / 2\right)}{Z_{0}(N, \beta)\left[\prod_{j=x, y, z} 2 \sinh \left(\beta \hbar w_{j} l / 2\right)\right]}\right)-\operatorname{coth}\left(\beta \hbar w_{i} / 2\right)\right]+\frac{N(N-1)}{2} \int d \mathbf{r} v_{2}(\mathbf{r}) g(\mathbf{r}) .
\end{aligned}
$$

In this expression, $Z_{0}(N, \beta)$ is the partition function of the model containing $N$ bosons at inverse temperature $\beta$ $=1 /\left(k_{B} T\right)$. In the isotropic case, $\Omega_{x}^{\prime}=\Omega_{y}^{\prime}=\Omega_{z}^{\prime}=\Omega^{\prime}$, and the parameters $\Omega^{\prime}$ and $w$ are the variational parameters. This gives a substantial simplification, since the variation with respect to $\Omega^{\prime}$ can be done analytically in the case of isotropy with $\Omega^{\prime}=\Omega$ as the result, i.e., the variational isotropic confinement frequency equals the isotropic confinement frequency of the examined system. However, for the anisotropic case, expression (17) has to be minimized with respect to all four parameters $\Omega_{x}^{\prime}, \Omega_{y}^{\prime}, \Omega_{z}^{\prime}$, and $\kappa$ to find the upper bound for the free energy.

\section{B. Variational free energy and condensation temperature for a Morse potential}

The variational free energy associated with the Morse potential is found using Eq. (17). The Morse potential appears from the interparticle interaction as the integral of the potential times the pair correlation function

$$
\begin{aligned}
& \frac{N(N-1)}{2} \int v_{2}(\mathbf{r}) g(\mathbf{r}) d \mathbf{r} \\
& \quad=\frac{N(N-1)}{2} U \int\left(e^{-2\left(r-r_{0}\right) / L}-2 e^{-\left(r-r_{0}\right) / L}\right) g(\mathbf{r}) d \mathbf{r} .
\end{aligned}
$$

Denoting $P_{l j}=\left(1-b^{j}\right)\left(1-b^{l-j}\right) /\left(1-b^{l}\right), \quad b=\exp \{-\beta \hbar w\}$, and $a_{w}=\sqrt{\hbar / m w}$, and using the Fourier transform of the pair correlation function, given in the Appendix, we find for the pair correlation function in the isotropic case 


$$
\begin{aligned}
N(N-1) g(\mathbf{r})= & \sum_{l=2}^{N} \frac{Z_{0}(N-l, \beta) b^{3 l / 2}}{Z_{0}(N, \beta)\left(1-b^{l}\right)^{3}} \sum_{j=1}^{l-1}\left[2 \pi a_{w}^{2} P_{l j}(b)\right]^{-3 / 2} \\
& \times\left[\exp \left(-\frac{r^{2}}{2 a_{w}^{2}} \frac{1}{P_{l j}(b)}\right)+\exp \left(-\frac{r^{2}}{2 a_{w}^{2}} P_{l j}(b)\right)\right],
\end{aligned}
$$

where $Z_{0}(N, \beta)$ is again the partition function of the model system. Details on the anisotropic pair correlation function are given in the Appendix. In this section, we assume isotropy in order not to complicate the formulas unnecessarily. Expression (18) reduces to

$$
\begin{aligned}
\frac{N(N-1)}{2} \int v_{2}(\mathbf{r}) g(\mathbf{r}) d \mathbf{r}= & \frac{U}{2 \sqrt{2 \pi}} \sum_{l=2}^{N} \frac{Z_{0}(N-l, \beta) b^{3 / 2}}{Z_{0}(N, \beta)\left(1-b^{l}\right)^{3}} \sum_{j=1}^{l-1}\left\{e^{2 r_{0} / L} f\left(2 P_{l j}^{1 / 2} \frac{a_{w}}{L}\right)-2 e^{r_{0} / L} f\left(P_{l j}^{1 / 2} \frac{a_{w}}{L}\right)\right. \\
& \left.+P_{l j}^{-3}\left[e^{2 r_{0} / L} f\left(2 P_{l j}^{-1 / 2} \frac{a_{w}}{L}\right)-2 e^{r_{0} / L} f\left(P_{l j}^{-1 / 2} \frac{a_{w}}{L}\right)\right]\right\},
\end{aligned}
$$

where $f$ is the following function of a dimensionless argument:

$$
f(x)=\sqrt{\frac{\pi}{2}}[1-\operatorname{erf}(x / \sqrt{2})]\left(x^{2}+1\right) e^{x^{2} / 2}-x .
$$

The expression for the variational free energy of this system is then given by substituting (20) in (17). In the isotropic case, one finds

$$
\begin{aligned}
F_{\text {Morse }} \leqslant & -\frac{\ln \left[Z_{0}(N, \beta)\right]}{\beta}+\left\{\frac{3}{\beta} \ln \left(\frac{\sinh (\hbar \beta \Omega / 2)}{\sinh (\hbar \beta w / 2)}\right)\right\}+\frac{3 \hbar\left[\Omega^{2}-w^{2}\right]}{4 w}\left[\left(\sum_{l=1}^{N} \frac{Z_{0}(N-l, \beta) \operatorname{coth}(\beta \hbar w l / 2)}{Z_{0}(N, \beta)[2 \sinh (\beta \hbar w l / 2)]^{3}}\right)-\operatorname{coth}(\beta \hbar w / 2)\right] \\
& +\frac{N(N-1)}{2} \frac{U}{\sqrt{2 \pi}} \sum_{l=2}^{N} \frac{Z_{0}(N-l, \beta) b^{3 l / 2}}{Z_{0}(N, \beta)\left(1-b^{l}\right)^{3}} \sum_{j=1}^{l-1}\left\{\begin{array}{c}
e^{2 r_{0} / L} f\left(2 P_{l j}^{1 / 2} \frac{a_{w}}{L}\right)-2 e^{r_{0} / L} f\left(P_{l j}^{1 / 2} \frac{a_{w}}{L}\right) \\
+P_{l j}^{-3}\left[e^{2 r_{0} / L} f\left(2 P_{l j}^{-1 / 2} \frac{a_{w}}{L}\right)-2 e^{r_{0} / L} f\left(P_{l j}^{-1 / 2} \frac{a_{w}}{L}\right)\right]
\end{array}\right\},
\end{aligned}
$$

where $\Omega$ equals the experimental confinement frequency and $w$ is the remaining variational parameter and again $a_{w}$ $=\sqrt{\hbar / m w}$. Details for the anisotropic model can be found in the Appendix. In this inequality, the expression obtained in [1] for $\left\langle\sum_{j=1}^{N} r_{j}^{2}\right\rangle$ has been used.

\section{RESULTS}

The variational free energy as a function of $w$ for a system with positive scattering length differs substantially from the case of negative scattering length. Therefore these cases are discussed separately.

\section{A. Morse potentials with positive scattering length}

For Morse potentials with positive scattering length, $F_{\text {Morse }}(w)$ has only one minimum. This minimum is located in $0<w<\Omega$ and the minimum value of the free energy at low temperatures is of the order of ground-state energy of the harmonic confinement potential. The variational free energy can be used to derive the condensation temperature of the Bose gas. For this purpose, the specific heat is calculated from the free energy $\left(c=-T \partial^{2} F / \partial T^{2}\right)$. For Morse poten- tials with a positive scattering length, a peak appears in the specific heat as a function of temperature, indicating the onset of Bose-Einstein condensation. We define the condensation temperature as the temperature at which the specific heat reaches its maximum. In the presence of interactions, the condensation temperature $T_{c}$ will differ from the condensation temperature $T_{c}^{0}$ of the noninteracting Bose gas in the same confinement potential. This is illustrated in Fig. 1, showing the specific heat as a function of temperature for several values of the scattering length. The relative shift in the condensation temperature induced by the interaction is denoted by $\delta_{T}=\left(T_{c}-T_{c}^{0}\right) / T_{c}^{0}$.

Figure 2 shows the interaction-induced shift $\delta_{T}$ as a function of the scattering length of the Morse potential. Typical parameter values for the Morse potentials used in Fig. 2 are $U=33.36 \times 10^{9} \hbar \Omega, \quad r_{0}=1.328 \times 10^{-4} a_{H O}, \quad L=4.794$ $\times 10^{-5} a_{H O}$ with $a_{H O}=(\hbar / m \Omega)^{1 / 2}$. Adapting the range $r_{0}$ of this Morse potential allows us to change the scattering length and set it to the value one wishes to study. Examples of scattering lengths appearing in experiments $[27,28]$ are given in Table I. A set of Morse potentials with different scattering lengths $a_{\text {scat }}$ was constructed, and for each of these Morse potentials, the interaction-induced shift $\delta_{T}$ in the condensa- 


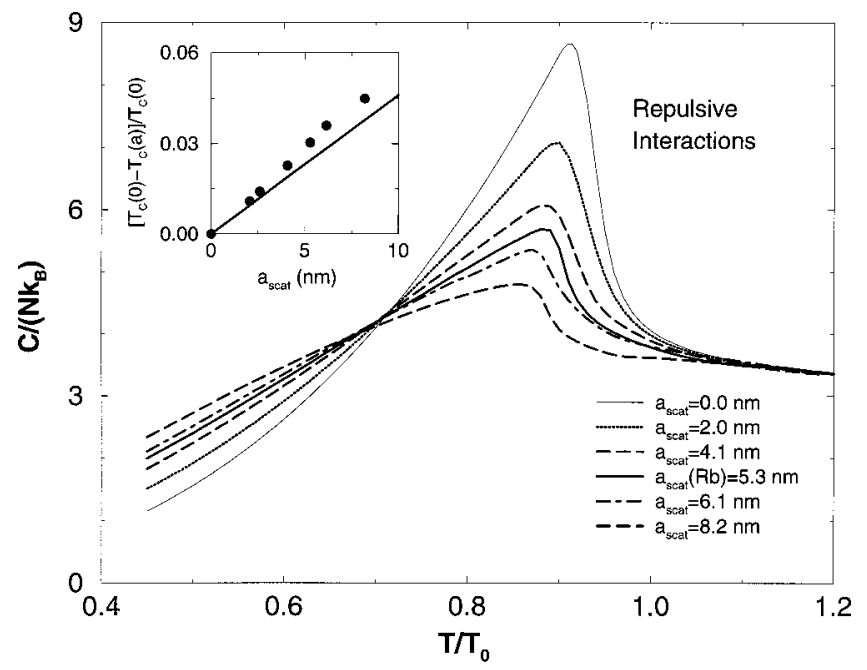

FIG. 1. The specific heat of a parabolically trapped Bose gas of 2000 atoms is shown as a function of temperature for several scattering lengths. In the inset the relative shift in the condensation temperature induced by the interparticle interaction is shown as a function of the scattering length. The condensation temperature is determined from the maximum of the specific heat. The full line is derived using Gross-Pitaevskii theory [9], the filled circles are obtained using the approach presented here.

tion temperature was calculated. The results are shown in Fig. 2 as the full circles. It should be noted that the condensation temperature of an ideal, trapped Bose gas and the condensation temperature of a Bose gas interacting through a Morse potential with zero scattering length coincide. The full line in Fig. 2 shows the predicted shift in condensation temperature for a contact potential, as obtained from solving the Gross-Pitaevski equation [9]. The results for the contact potential and the Morse potential approach each other for small scattering length. One could also adapt the scattering length of the Morse potential by adapting the parameter $U$. Choosing $U$ instead of $r_{0}$ as the parameter to adjust the scattering length had no noticeable effect on the interaction-induced shift in the condensation temperature. In the inset, the optimal value of the variational parameter $w$ is shown as a function of temperature. For a repulsive Morse potential ( $a_{\text {scat }} / a_{\mathrm{HO}}>0$, full line) we find that the size of the atom

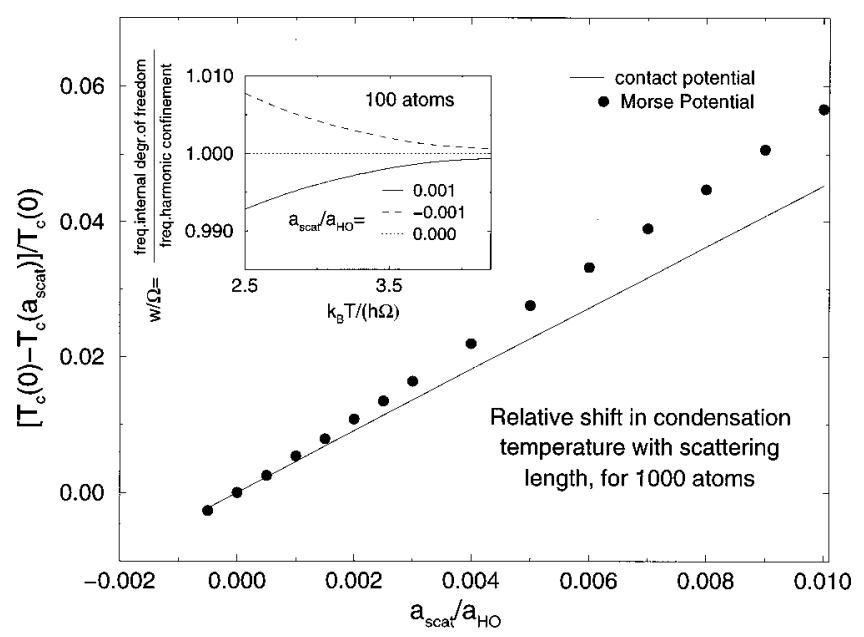

FIG. 2. The interaction-induced shift of the condensation temperature is shown as a function of the interaction strength $a_{\text {scat }} / a_{H O}$ for Morse potentials (filled circles, calculated in the present approach) and contact potentials (full line, calculated from the Gross-Pitaevskii equation [9]). In the inset, the variational optimal value for the parameter $w / \Omega$ is shown as a function of temperature, for a repulsive (full line) and an attractive (dashed line) Morse potential and for the noninteracting, parabolically trapped Bose gas (dotted line).

cloud is expanded since $w<\Omega$. An attractive Morse potential ( $a_{\text {scat }}<0$, discussed below, dashed line in the inset), on the other hand, will contract the gas.

\section{B. Morse potentials with negative scattering length}

For Morse potentials with negative scattering length, $F_{\text {Morse }}(w)$ has two minima separated by a free energy barrier. There is again a minimum for $w$ of the order of $\Omega$, but a second minimum is found at a much higher frequency near $w \approx \Omega\left(a_{H O} / r_{0}\right)^{2} \gg \Omega$, where $r_{0}$ reflects the range of the attractive part of the interaction and $a_{H O}=\sqrt{\hbar / m \Omega}$. This second minimum has a free energy value of the order of $-N U$, where $U$ reflects the depth of the attractive part of the interatomic potential. The average distance between the bosons is of the order of $(\hbar / m w)^{-1 / 2}$ and is thus comparable to the range of the interatomic potential. Hence, it is plau-

TABLE I. Typical values for system parameters in experiments on ultracold Bose gases. For a set of chosen alkali atoms we list the scattering lengths $a_{\text {scat }}$ (taken from [27]), the frequencies of the parabolic confinement potential (taken from [28]), typical number of atoms for which Bose-Einstein condensation is observed (where applicable), the effective interaction strength given by $a_{\text {scat }} / a_{H O}$, and the critical number $N_{c}$ (found by the present method) at zero temperature (only applicable for negative scattering lengths). n.a. stands for not applicable.

\begin{tabular}{|c|c|c|c|c|c|}
\hline Atom & $a_{\text {scat }}$ & $N$ & $\tilde{\Omega}=\sqrt{3} \Omega_{x} \Omega_{y} \Omega_{z}$ & $a_{\text {scat }} / a_{\mathrm{HO}}$ & $N_{c}$ \\
\hline${ }^{7} \mathrm{Li}$ & $-1.44 \pm 0.04 \mathrm{~nm}$ & 1300 & $144 \mathrm{~Hz}$ & $-4.64 \times 10^{-4}$ & 1443 \\
\hline${ }^{23} \mathrm{Na},|1,-1\rangle$ & $4.9 \pm 1.4 \mathrm{~nm}$ & 500000 & $416 \mathrm{~Hz}$ & 0.0048 & n.a. \\
\hline${ }^{87} \mathrm{Rb}$ & $4.6 \pm 1.1 \mathrm{~nm}$ & 4500 & $187 \mathrm{~Hz}$ & 0.0058 & n.a. \\
\hline${ }^{85} \mathrm{Rb}$ & $-50 \mathrm{~nm}<\mathrm{a}_{\text {scat }}<-3 \mathrm{~nm}$ & n.a. & $\sim_{1} \mathrm{~Hz}$ & -0.0050 & 134 \\
\hline${ }^{133} \mathrm{Cs}$ & $-13 \mathrm{~nm}$ & n.a. & $34 \mathrm{~Hz}$ & -0.0087 & 77 \\
\hline
\end{tabular}


sible that the second minimum in the free energy, which appears for Bose gases with negative scattering length, corresponds to a many-particle bound state. We will refer to this state as the "clustered" state, and to the state corresponding to the minimum with $w$ of the order of $\Omega$ as the "gaseous" state. Since the free energy in the clustered state is lower than the free energy in the gaseous state, the latter is metastable with respect to a transition to the clustered state. When the scattering length is not negative, the minimum in the free energy at a large ("cluster") value of $w$ is not present. This property of the free energy has been checked numerically for a Morse potential and has been obtained analytically for any two-step square well potential with a non-negative scattering length.

For the negative scattering lengths, only the specific heat associated with the gaseous state shows a peak as a function of temperature, and thus we find a condensation temperature only in the gaseous state, as expected. The interactioninduced shift in the condensation temperature is opposite to the shift for potentials with positive scattering length, as shown in Fig. 2.

\section{Phase diagram for a Bose gas with negative scattering length}

As discussed above for the Morse potentials with negative scattering length, there are in general two minima in the free energy. However, when the number of particles is increased at fixed temperature, we find that the minimum in the variational free energy associated with the gaseous state disappears above a critical number $N_{c}$ of particles. The analytic expression for this critical number at temperature zero can be found using that $Z_{0}(N-l, \beta) b^{3 l / 2} / Z_{0}(N, \beta) \rightarrow 1$ and $P_{l j} \rightarrow 0$ for $T \rightarrow 0$, such that

$$
\begin{aligned}
F_{\text {Morse }}(T \rightarrow 0)= & \frac{3}{2} \hbar w(N-1)+\frac{3}{2} \hbar \Omega \\
& +\frac{3 \hbar\left(\Omega^{2}-w^{2}\right)}{4 w}(N-1) \\
& +\frac{N(N-1)}{\sqrt{2 \pi}} U e^{r_{0} / L}\left[e^{r_{0} / L} f\left(2 a_{w} / L\right)\right. \\
& \left.-2 f\left(a_{w} / L\right)\right] .
\end{aligned}
$$

Then $N_{c}(T \rightarrow 0)$ can be found by treating the variational equation $\partial F / \partial w=0$ as an equation in $w$ and $N$ and finding the maximal $N$ possible as a function of $w$. In the gaseous state $a_{w} / r_{0} \gg 1$ and the asymptotic form of $f$ can be used, yielding

$$
N_{c}(T \rightarrow 0)=\frac{\sqrt{8 \pi}}{5^{5 / 4}} \frac{a_{H O}}{L} \frac{\hbar^{2} /\left(m L^{2}\right)}{U} e^{-r_{0} / L}\left(\frac{1}{4}-\frac{4}{e^{r_{0} / L}}\right),
$$

with again $a_{H O}=\sqrt{\hbar / m \Omega}$. The relation of the Morse potential parameters to the scattering length $a_{\text {scat }}$ of the potential allows us to rewrite Eq. (24) as $N_{c}(T \rightarrow 0)=$ $-\left(\sqrt{8 \pi} / 5^{5 / 4}\right) a_{H O} / a_{\text {scat }} \approx-0.67 a_{H O} / a_{\text {scat }}$. This theoretical value for $N_{c}$ in the limiting case of $T \rightarrow 0$ was also obtained

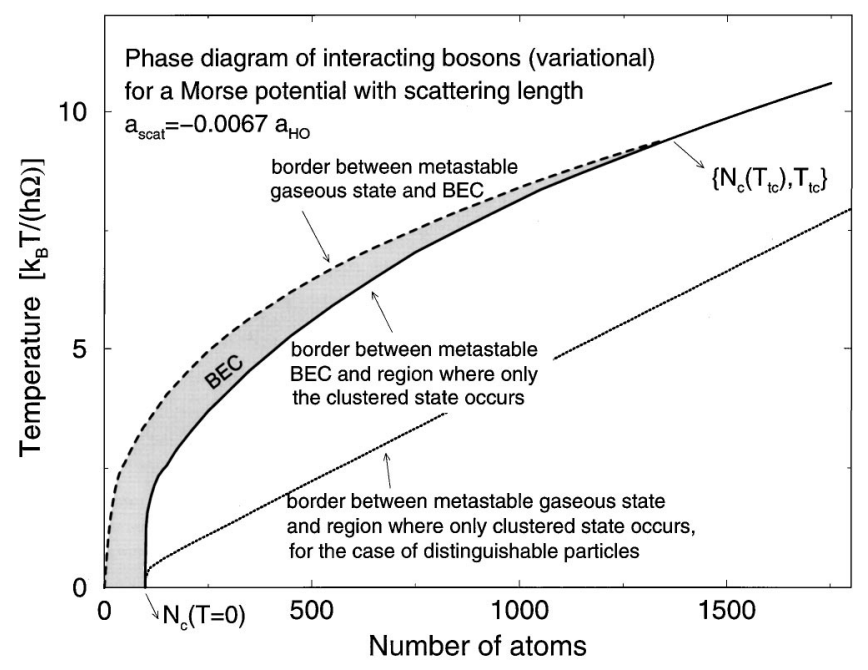

FIG. 3. A $(N, T)$ phase diagram is shown for a gas of bosons interacting through a Morse potential with negative scattering length $a_{\text {scat }} / a_{H O}=-0.0067$ with $a_{H O}=\sqrt{\hbar / m \Omega}$, such that $N_{c}(T$ $=0)=100$. The dashed line shows the condensation temperature $T_{c}$ as a function of the number of bosons. The full line shows the critical number of bosons $N_{c}$ beyond which the gaseous state no longer exists, as a function of temperature, and the dotted line shows this critical number for distinguishable particles under the same conditions. The tricritical temperature $T_{t c}$, above which BEC is not possible regardless of the number of bosons, is indicated: $T_{t c}=9.43 \hbar \Omega / k_{B}, N_{c}\left(T_{t c}\right)=1363$.

by Salasnic [29] using a Gaussian variational wave function and the Rayleigh-Ritz variational principle. The advantage of the present method, however, is that unlike a variational approach based on a trial wave function, the current technique allows us to calculate $N_{c}$ at any temperature. The temperature dependence of the critical number $N_{c}(T)$ is shown in Fig. 3. Figure 3 represents a phase diagram in the $N, T$ plane for the case of negative scattering length $a_{\text {scat }} / a_{H O}=-6.7$ $\times 10^{-3}$, a value typical for the experiments on ultracold trapped atoms (see also Table I). This specific choice for $a_{\text {scat }} / a_{H O}$ corresponds to $N_{c}=100$ at zero temperature. Several regions can be distinguished in this phase diagram: a region where the metastable gaseous state exists and is not Bose condensed, a region where the metastable gaseous state exists as Bose condensate, and finally a region where only the clustered state was found by the present approach.

Keeping the temperature $T$ fixed, we find a number of particles $N_{b}(T)$ such that Bose-Einstein condensation sets in for $N_{b}(T)<N$. We also find the critical number of particles $N_{c}(T)$ above which Bose-Einstein condensation is no longer possible. As long as $N_{b}(T)<N_{c}(T)$, Bose-Einstein condensation can be realized at the given temperature $T$ for $N_{b}(T)$ $<N<N_{c}(T)$. As the temperature is increased, $N_{b}(T)$ $\rightarrow N_{c}(T)$ until at the tricritical temperature $T_{t c}$ (indicated in Fig. 2) one has $N_{b}\left(T_{t c}\right)=N_{c}\left(T_{t c}\right)$. For temperatures above this tricritical temperature, Bose-Einstein condensation is no longer possible, regardless of the number of particles in the gas.

Keeping the number of particles $N$ fixed, we find a condensation temperature $T_{c}(N)$ but also a temperature $T_{c l}(N)$ 


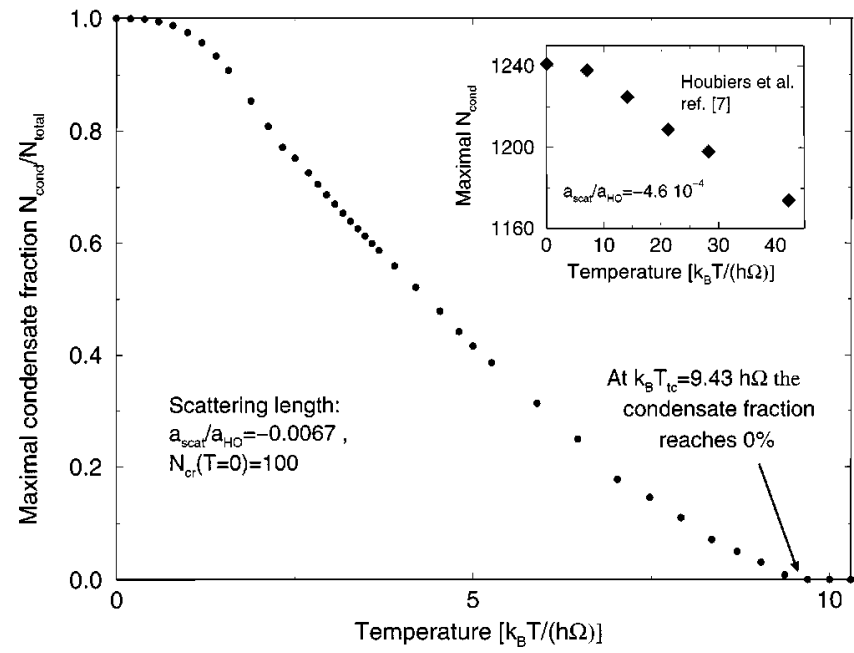

FIG. 4. $N_{c}$ is the maximum number of atoms in the trap that can undergo Bose-Einstein condensation. Using this number, and the associated condensation temperature $T_{c}\left(N_{c}\right)$ the maximal fraction of atoms in the condensate is shown as a function of temperature for a gas with scattering length $a_{\text {scat }} / a_{H O}=-6.7 \times 10^{-3}$. In the inset, the maximum occupation of the condensate calculated in [7] with a $T$-matrix approach and for a scattering length $a_{\text {scat }} / a_{H O}=-4.6$ $\times 10^{-4}$ is shown.

such that for temperatures lower than $T_{c l}(N)$ only the clustered state is found by the present approach. Thus, BoseEinstein condensation for a given total number of particles $N$ (both condensate and noncondensate) can only occur in a temperature range $T_{c l}(N)<T<T_{c}(N)$. This means that, given a fixed total number of particles, the gas can only be cooled to a temperature $T_{c l}(N)$, and not to $T=0$. Since the gas has to be cooled all the way down to $T=0$ in order to have a condensate fraction of $100 \%$, and since it can only be cooled to $T_{c l}(N)$, the maximal condensate fraction of a Bose gas with negative scattering length will be less than $100 \%$ for $N$ such that $T_{c l}(N)>0$.

In our approach the maximum total number of bosons $N_{c}(T)$ in the trap at a given temperature is obtained. This number can be used to estimate the fraction of the atoms in the condensate using $1-\left\{T / T_{c}\left[N_{c}(T)\right]\right\}^{3}$, where $T_{c}(N)$ denotes the condensation temperature for $N$ bosons. The condensate fraction can then be studied as a function of the temperature for the scattering length used in the preceeding calculation, $a_{\text {scat }} / a_{H O}=-6.7 \times 10^{-3}$. The temperature dependence of this condensate fraction is shown in Fig. 4. In the inset, the number of atoms in the condensate, calculated in Ref. [7] within the $T$-matrix approach but for a different scattering length $a_{\text {scat }} / a_{H O}=-4.64 \times 10^{-4}$, is shown for comparison. Note that the decrease in the condensate fraction is much less pronounced in the case of Houbiers and Stoof [7]. This may be due to the fact that in the present approach the thermodynamical properties are calculated as a function of the total number of atoms in the trapped gas, both condensed and noncondensed. Houbiers and Stoof, on the other hand, calculated the number of condensed atoms in the grand canonical ensemble, given a nonconserved density as a reservoir.
As a final remark, we wish to point out that the variational method presented in Sec. II can also be applied to a gas of distinguishable particles (without Bose statistics). In this manner, the effects of statistics on, for example, the clustering, can be studied. For distinguishable particles, no sums over permutations must be taken, and the expression for the variational free energy (in isotropic confinement) simplifies to

$$
\begin{aligned}
F_{\text {Morse }}^{\text {dist. }} \leqslant & -\frac{1}{\beta} \ln \left(\frac{[2 \sinh (\beta \hbar \Omega / 2)]^{-3}}{[2 \sinh (\beta \hbar w / 2)]^{3(N-1)}}\right) \\
& +\frac{3 \hbar\left(\Omega_{0}^{2}-w^{2}\right)(N-1)}{4} \frac{\operatorname{coth}(\hbar \beta w / 2)}{w} \\
& +\frac{3 \hbar\left(\Omega_{0}^{2}-\Omega^{2}\right)}{4} \frac{\operatorname{coth}(\beta \hbar \Omega / 2)}{\Omega}+\frac{U N(N-1)}{(2 \pi)^{1 / 2}} \\
& \times\left[-2 f\left(a_{T} / L\right) e^{r_{0} / L}+f\left(2 a_{T} / L\right) e^{2 r_{0} / L}\right],
\end{aligned}
$$

where $a_{T}=a_{w} \sqrt{\operatorname{coth}(\hbar \beta w / 2)}$, and $\Omega_{0}$ is the experimental confinement frequency. The variational free energy of the system of distinguishable particles no longer gives rise to a peak in the specific heat as a function of temperature, indicating that there is no phase transition to an ordered state (the condensate). As it should be, no condensation temperature $T_{c}>0$ is found. But for Morse potentials with negative scattering length, the variational free energy will again allow for a clustered state with respect to which the gaseous state is metastable. One may wonder whether the critical number of particles above which the present approach only finds the clustered state depends on the Bose statistics: is it the same for distinguishable particles as for bosons? The answer, as given by the present calculation, is no: the critical number of particles $N_{c}^{d}(T)$ for the distinguishable particles, shown as the dotted line in Fig. 3, differs from the critical number of particles $N_{c}(T)$ for the bosons except at $T=0$. At a given temperature, the critical number above which only the clustered state persists is lower for bosons than for distinguishable particles under the same conditions. For temperatures approaching zero, the critical number for bosons approaches that for distinguishable particles.

\section{Case study: The lithium condensate}

In contrast to a standard variational approach using a trial wave function, there is no simple criterion to estimate how close the variational upper bound for the free energy lies to the real value of the free energy of the system. In order to check the robustness of the variational estimate for the free energy, we focus on a case study for lithium. Instead of using the Morse potential, as in the previous subsections, we use the real interaction potential, experimentally derived from spectroscopy measurements [20-22], and compare the results for this potential with the analytical results found for the Morse potential. 
TABLE II. Results of the variation for the free energy at temperature zero, in units $m_{\mathrm{Li}}=\hbar=\Omega=1$. The first column shows the number of particles in the parabolic confinement. The next columns show the optimal variational value for the parameter $w$ and the variational free energy for (i) the gaseous state (identical results were found for the Morse potential and the real potential), (ii) the Morse potential, and (iii) the experimentally derived triplet potential [20-22]. Comments are added in the last column.

\begin{tabular}{lccccccc}
\hline \hline & Gaseous & & Clustered & (Morse) \\
$N$ & $w_{1}$ & $F_{1} / N$ & $w_{2, \text { Morse }}$ & $F_{2, \text { Morse }} / N$ & $\begin{array}{c}\text { Clustered } \\
w_{2, \mathrm{Li}}\end{array}$ & $\begin{array}{c}\text { (exact) } \\
F_{2, \mathrm{Li}} / N\end{array}$ & Comment \\
\hline 2 & 1.00037 & 1.49981 & no minimum & & no minimum & & only \\
5 & 1.00091 & 1.49926 & no minimum & & no minimum & & gaseous \\
10 & 1.00184 & 1.49833 & no minimum & & no minimum & & state \\
20 & 1.00373 & 1.49647 & no minimum & & no minimum & & \\
21 & 1.00392 & 1.49628 & 53389.8 & 4714.57 & 57555.7 & 4268.57 & clustered \\
22 & 1.00411 & 1.49610 & 60304.0 & 3005.24 & 63920.3 & 2406.39 & state is \\
23 & 1.00430 & 1.49591 & 65178.6 & 1100.04 & 68640.5 & 357.693 & metastable \\
24 & 1.00449 & 1.49572 & 69042.2 & -948.329 & 72454.2 & -1831.68 & gaseous \\
100 & 1.01925 & 1.48140 & 111411 & $-2.21442 \times 10^{5}$ & 115733 & $-2.34133 \times 10^{5}$ & state is \\
1000 & 1.31950 & 1.27743 & 120331 & $-3.01629 \times 10^{6}$ & 124993 & $-3.17466 \times 10^{6}$ & metastable \\
1443 & 2.16973 & 1.11880 & 120623 & $-4.39386 \times 10^{6}$ & 125297 & $-4.62399 \times 10^{6}$ & \\
1444 & no minimum & & 120624 & $-4.39697 \times 10^{6}$ & 125298 & $-4.62726 \times 10^{6}$ & only clustered \\
1500 & no minimum & & 120648 & $-4.57111 \times 10^{6}$ & 125323 & $-4.81048 \times 10^{6}$ & state \\
\hline \hline
\end{tabular}

This implies that we have to first describe how we used the real interaction potential in the calculation and second how we obtained the parameters of the Morse potential for these atoms. For distances less than $0.15 \mathrm{~nm}$, the core region, the real interaction potential is not determined experimentally. We introduced a constant potential $V_{\text {core }}$ that is adjusted to the known scattering length [20-22]. Anticipating on the results, it should be noted that the introduction of a constant potential does not strongly influence the results. One of the reasons may be that the region of the core is small compared to the region covered by a substantial value of the pair correlation. The remaining experimental parameters of the trap, such as the confinement frequency, were chosen in agreement with the experimental setup of [30].

The Morse potential (1) for the lithium triplet interaction is obtained as described before. The scattering length is fixed to its experimental value $(-27.6 \mathrm{~nm})$ and the number of bound levels $(11,[31])$ is introduced. The remaining parameters are determined by a least-squares fit to the measured triplet potential, leading to $U=33.002 \times 10^{9} \hbar \Omega, \quad r_{0}$ $=1.2531 \times 10^{-4} a_{\mathrm{HO}}, L=4.5236 \times 10^{-5} a_{\mathrm{HO}}$.

The results at zero temperature are summarized in Table II. For the variational free energy in the gaseous state, no differences were found in the results for the Morse potential and for the experimental potential. The variational free energy in the gaseous state, and the value of $w$ associated with it, are listed in columns 2 and 3 of Table II. In the next two columns, the free energy and the associated $w$ are shown for the clustered state for the Morse potential. Columns 5 and 6 show the free energy and $w$ for the clustered state for the experimental potential. Note that at the lowest particle numbers, the free energy of the clustered state is increased, and even becomes larger than the free energy of the gaseous state. This result deserves further attention, but in our opinion it is a typical few-body problem where the molecular potentials have to be taken into account to higher orders than we did here. Therefore we expect that the model system is no longer adequate for such a small number of particles.

The agreement between the clustered state for the Morse potential and for the experimental potential is good, and increases in accuracy as the number of particles increases. Also the critical number $N_{c}$ remains the same. The result for the critical number of particles for the experimental setup of [30], with scattering length and trapping parameters such that $a_{\text {scat }} / a_{H O}=4.64 \times 10^{-4}$, is $N_{c}=1443$ in the present approach. This number $N_{c}$ represents the maximum number of atoms in the trap that still can undergo Bose-Einstein condensation. Thus it is an upper bound to the average number of atoms that is reported in [30] to lie between 600-1300.

\section{DISCUSSION AND CONCLUSION}

The method proposed in this paper allows us to incorporate any two-body interaction $v_{2}$ in the description of the thermodynamics of an interacting Bose gas. From this point of view, its application is more general than the GrossPitaevskii model, which takes into account a two-body contact potential $v_{\delta}(\mathbf{r})=4 \pi \delta(\mathbf{r}) \hbar^{2} a_{\text {scat }} / m$ with scattering length $a_{\text {scat }}$, and which hence neglects the range and shape of the interatomic potential. From the expression of the expectation value of the two-body interaction in the JensenFeynman inequality, it is clear that this approximation will be valid as long as the range of the interatomic potential (of the order of $r_{0}$ for the Morse potential used above; see Table III) is much shorter than the typical length scale $\xi$ over which the pair correlation function varies. Indeed, in that case, the expectation value of the interatomic potential essentially depends on the value of the pair correlation function in the origin and on the scattering length: 
TABLE III. A description of the different length scales used in the paper is given.

\begin{tabular}{lc}
\hline \hline Symbol & Description \\
\hline$a_{\text {scat }}$ & scattering length \\
$a_{H O}$ & $\sqrt{\hbar /(m \Omega)}$ \\
$a_{w}$ & $\sqrt{\hbar /(m w)}$ \\
$a_{T}$ & $a_{w} \sqrt{\operatorname{coth}\left[\hbar w /\left(2 k_{B} T\right)\right]}$ \\
$r_{0}$ & distance at which the Morse potential \\
& reaches its minimum \\
\hline
\end{tabular}

$$
\begin{aligned}
\left\langle v_{2}\right\rangle & =\frac{N(N-1)}{2} \int d \mathbf{r} g(\mathbf{r}) v_{2}(\mathbf{r}) \rightarrow\left\langle v_{2}\right\rangle_{r_{0} \ll \xi} \\
& \approx \frac{N(N-1)}{2} g(0) \int d \mathbf{r} v_{2}(\mathbf{r}) \\
& =\frac{2 \pi \hbar^{2} a_{\text {scat }}}{m} N(N-1) g(0) .
\end{aligned}
$$

However, even for short-range interactions, the typical length scale of the pair correlation function can become comparable to the range of the interatomic interactions. This happens for interatomic potentials that allow for a clustered state (a bound state involving many particles). In that case, the details of the interaction potential become important and models based on the Gross-Pitaevskii equation using a contact potential lead to an unphysical result: the free energy in these models is not bound from below. This artifact is avoided in the present treatment by taking into account the range of the interatomic potential.

Bose-Einstein condensation in a gas with negative scattering length has been achieved experimentally: for a parabolically confined gas of lithium atoms a Bose-Einstein condensate of at most 600-1300 bosons [30] was observed, comparable to the critical number for this experiment, predicted from the theory presented here. It should be noted that the present analysis does not describe the dynamics of a cooling Bose gas nor does it describe the dynamics of the formation of the clustered state. The critical number of bosons beyond which Bose-Einstein condensation is no longer possible may be different in the experiment due to the dynamical effects.

In the theoretical approach presented here, as well as in the two-fluid models [9-13] for the condensate and even in the Monte Carlo simulations [32,33], it is tacitly assumed that the system is in thermal equilibrium. It is also clear that the measurements are done on systems that are not in equilibrium at all; even obtaining a steady state seems at present out of experimental reach $[34,35]$. This means that in discussing the theoretical results we rely on our intuition of how the calculated equilibrium situation is reached without conflicting with the known experimental facts for the nonequilibrium situation. For our approach, this means that we have to argue how it is possible that a metastable state with a much higher energy than the ground state can be studied experimentally, while the ground state itself does not even show up circumstantially. For distinguishable particles it is generally accepted that the lifetime of a metastable state is proportional to the energy barrier between the two states and inversely proportional to their energy difference. There are different scenarios possible. The first one implicates gravity: as soon as a cluster of a few atoms is formed it leaves the trap due to the gravitational force, thereby reducing the number of atoms available for condensation and increasing the average energy present in the trap, because the low-energy states can escape now. In this scenario one is allowed to start with a number of atoms larger than the calculated $N_{c}$. After some time of operation, clustering will reduce the number of atoms in the trap. Once the number of atoms is lower, the metastable state becomes available as the first excited state of the system. If the collision rate in this state does not differ fundamentally from the collision rate of the previous situation, the clustering will go on, and eventually all particles will have left the trap. If there is a change in collision rate, or if the state is almost collision free, the metastable state can get the necessary lifetime to be available for experimentation. For ${ }^{87} \mathrm{Rb}$ this change in collision rate was found [36] to be due to the influence of the condensate on the loss rate of three-body recombinations. This characteristic was predicted by Kagan et al. [37] who also suggest its use as the signature of the presence of the condensate. The physical origin of the change in collision rate is due to a change in distribution. While in the high-temperature phase the distribution is Gaussian (at least to a good approximation) it would remain Gaussian (with different parameters) if the particles were distinguishable. Projecting it on the symmetric irreducible representation of the permutation group as required for indistinguishable particles, the distribution takes the equilibrium of the condensate and its excitations into account.

In this paper, we presented a path-integral variational method which (1) exactly treats the quantum statistics at any temperature and (2) allows the incorporation of finite-range two-body interactions in the description of the thermodynamics of the Bose gas. After presenting this technique, we focused on a gas of parabolically trapped bosonic atoms interacting through a Morse potential. The effect of this interaction on the condensation temperature was studied. For Morse potentials with a negative scattering length, a phase diagram was derived showing the influence of the interaction on the region in $N, T$ parameter space favorable to BoseEinstein condensation. Apart from the interaction-induced shift in condensation temperature, the region in $N, T$ parameter space available for Bose-Einstein condensation is reduced by the presence of a clustered phase. The effect of the interaction and the Bose statistics on the critical number of particles above which the gaseous state was no longer found in the present treatment was calculated. We identified a temperature above which Bose-Einstein condensation is no longer possible regardless of the number of particles.

\section{ACKNOWLEDGMENTS}

Discussions with S. Stringari, Y. Kagan, S. Giorgini, and L. Salasnic are gratefully acknowledged. We thank H. Stoof for discussions and for making the results on the ${ }^{7} \mathrm{Li}$ poten- 
tial available. This work was performed within the framework of the FWO Project Nos. 1.5.729.94, 1.5.545.98, G.0287.95, G.0071.98, and WO.073.94N (Wetenschappelijke Onderzoeksgemeenschap, Scientific Research Community of the FWO on Low Dimensional Systems), the "Interuniversitaire Attractiepolen - Belgische Staat, Diensten van de Eerste Minister - Wetenschappelijke, Technische en Culturele Aangelegenheden," and in the framework of the BOF NOI 1997 projects of the Universiteit Antwerpen. Two of the authors (J.T. and F.B.) acknowledge the FWO (Fonds voor Wetenschappelijk Onderzoek-Vlaanderen) for financial support.

\section{APPENDIX}

The expectation values of one-body and two-body potential energies appearing in the Jensen-Feynman inequality can be expressed as a function of the density

$$
\begin{aligned}
& \sum_{j=1}^{N} v_{1}\left(\mathbf{r}_{j}\right)=\int d \mathbf{r} v_{1}(\mathbf{r}) n(\mathbf{r}) \quad \text { with } \\
& n(\mathbf{r})=\int \frac{d \mathbf{k}}{(2 \pi)^{3}} e^{-i \mathbf{k} \cdot \mathbf{r}}\left\langle\sum_{j=1}^{N} e^{i \mathbf{k} \cdot \mathbf{r}_{j}}\right\rangle,
\end{aligned}
$$

and of the pair correlation function:

$$
\frac{1}{2} \sum_{j=1}^{N} \sum_{l=1}^{N} v_{2}\left(\mathbf{r}_{j}-\mathbf{r}_{l}\right) \Theta(l \neq j)=\frac{N(N-1)}{2} \int d \mathbf{r} v_{2}(\mathbf{r}) g(\mathbf{r})
$$

with

$$
\begin{aligned}
g(\mathbf{r})= & \frac{1}{N(N-1)} \int \frac{d \mathbf{k}}{(2 \pi)^{3}} \\
& \times e^{-i \mathbf{k} \cdot \mathbf{r}}\left\langle\sum_{j=1}^{N} \sum_{l=1}^{N} e^{i \mathbf{k} \cdot\left(\mathbf{r}_{j}-\mathbf{r}_{l}\right)} \Theta(l \neq j)\right\rangle .
\end{aligned}
$$

The symbol $\Theta($ expr $)=1$ if the logical expression expr is true, and zero otherwise. These quantities have been discussed in detail in [1]. In this appendix, the generalization of the results of [1] to the case of anisotropic confinement will be given. For a spin-polarized gas of bosons in an anisotropic parabolic confinement potential with frequencies $\Omega_{x}, \Omega_{y}, \Omega_{z}$ and a two-body potential energy $(\kappa / 4) \Sigma_{j, l}\left(\mathbf{r}_{j}-\mathbf{r}_{l}\right)^{2}$, the propagator is given by

$$
\begin{gathered}
K\left(\mathbf{r}_{1}, \ldots, \mathbf{r}_{N} ; \beta \mid \mathbf{r}_{1}, \ldots, \mathbf{r}_{N} ; 0\right) \\
=\frac{1}{N !} C(\mathbf{R}) \sum_{P} \prod_{j=1}^{N} K_{w_{x}, w_{y}, w_{z}}\left(P\left[\mathbf{r}_{j}\right] ; \beta \mid \mathbf{r}_{j} ; 0\right), \\
C(\mathbf{R})=\frac{K_{\Omega_{x}, \Omega_{y}, \Omega_{z}}(\sqrt{N} \mathbf{R} ; \beta \mid \sqrt{N} \mathbf{R} ; 0)}{K_{w_{x}, w_{y}, w_{z}}(\sqrt{N} \mathbf{R} ; \beta \mid \sqrt{N} \mathbf{R} ; 0)}
\end{gathered}
$$

where the first sum runs over all particle permutations $P$; $w_{i}=\left(\Omega_{i}^{2}-N \kappa\right)^{1 / 2}$ are the renormalized frequencies; $\mathbf{R}$ $=(1 / N) \Sigma_{j} \mathbf{r}_{j}$ is the center-of-mass coordinate; and $K_{v_{x}, v_{y}, v_{z}}$ is the single-particle path-integral propagator for an anisotropic harmonic oscillator with frequencies $v_{x}, v_{y}, v_{z}$. Hence

$$
\begin{aligned}
Z_{0}(N, \beta)= & \sum_{j=1}^{N} \int d \mathbf{r}_{1} \cdots d \mathbf{r}_{N} C(\mathbf{R}) \frac{1}{N !} \\
& \times \sum_{P}\left[\prod_{v=1}^{N} K_{w_{x}, w_{y}, w_{z}}\left(P\left[\mathbf{r}_{v}\right] ; \beta \mid \mathbf{r}_{v} ; 0\right)\right], \\
\left\langle\sum_{j=1}^{N} e^{\left.i \mathbf{k} \cdot \mathbf{r}_{j}\right\rangle=}\right. & \frac{1}{Z_{0}(N, \beta)} \sum_{j=1}^{N} \int d \mathbf{r}_{1} \cdots d \mathbf{r}_{N} \\
& \times C(\mathbf{R}) \frac{1}{N !} \sum_{P}\left[\prod_{v=1}^{N}\right. \\
& \left.\times K_{w_{x}, w_{y}, w_{z}}\left(P\left[\mathbf{r}_{v}\right] ; \beta \mid \mathbf{r}_{v} ; 0\right) e^{i \mathbf{k} \cdot \mathbf{r}_{j}}\right],
\end{aligned}
$$

$$
\begin{aligned}
\left\langle\sum_{j=1}^{N}\right. & \left.\sum_{l=1}^{N} e^{i \mathbf{k}\left(\mathbf{r}_{j}-\mathbf{r}_{l}\right)} \Theta(l \neq j)\right\rangle \\
= & \frac{1}{Z_{0}(N, \beta)} \sum_{j=1}^{N} \sum_{l=1}^{N} \Theta(l \neq j) \int d \mathbf{r}_{1} \cdots d \mathbf{r}_{N} C(\mathbf{R}) \frac{1}{N !} \\
& \times \sum_{P}\left[\prod_{v=1}^{N} K_{w_{x}, w_{y}, w_{z}}\left(P\left[\mathbf{r}_{v}\right] ; \beta \mid \mathbf{r}_{v} ; 0\right) e^{i \mathbf{k}\left(\mathbf{r}_{j}-\mathbf{r}_{l}\right)}\right],
\end{aligned}
$$

where $Z_{0}(N, \beta)$ indicates the partition function in the analytical model system. Each permutation can be written as a cyclic decomposition with $M_{1}$ cycles of length $1, M_{2}$ cycles of length $2, \ldots$. The sum over permutations can be transformed into a sum over cyclic decompositions, as in Feynman's treatment of the ideal homogeneous Bose gas [25]:

$$
\sum_{P} \rightarrow \sum_{\left\{M_{1}, M_{2}, \ldots\right\}} N ! \prod_{l} \frac{1}{M_{l} ! l^{M_{l}}} \Theta\left(\sum_{l} l M_{l}=N\right) .
$$

The constraint in the cyclic sum appears in order to guarantee that the total number of elements in all cycles of the cyclic decomposition equals the total number of bosons in the gas. For example, the partition sum becomes

$$
\begin{aligned}
Z_{0}(N, \beta)= & \sum_{j=1}^{N} \int d \mathbf{r}_{1} \cdots d \mathbf{r}_{N} C(\mathbf{R}) \\
& \times \sum_{\left\{M_{1}, M_{2}, \ldots\right\}}\left[\prod_{l} \frac{1}{M_{l} ! l^{M_{l}}} K_{l}^{M_{l}}\right] \\
& \times \Theta\left(\sum_{l} l M_{l}=N\right),
\end{aligned}
$$




$$
\begin{aligned}
K_{l}= & K_{w_{x}, w_{y}, w_{z}}\left(\mathbf{r}_{1} ; \beta \mid \mathbf{r}_{l} ; 0\right) \cdots K_{w_{x}, w_{y}, w_{z}}\left(\mathbf{r}_{3} ; \beta \mid \mathbf{r}_{2} ; 0\right) \\
& \times K_{w_{x}, w_{y}, w_{z}}\left(\mathbf{r}_{2} ; \beta \mid \mathbf{r}_{1} ; 0\right) .
\end{aligned}
$$

The restriction $\Sigma_{l} l M_{l}=N$ is prohibitive for performing the summation directly. To lift this restriction, and perform the summation, the generating functions are introduced:

$$
\begin{gathered}
\Xi(u)=\sum_{N=0}^{\infty} Z_{0}(N, \beta) u^{N}, \\
G_{1}(\mathbf{k}, u)=\sum_{N=0}^{\infty} Z_{0}(N, \beta)\left\langle\sum_{j=1}^{N} e^{i \mathbf{k} \cdot \mathbf{r}_{j}}\right\rangle u^{N}, \\
G_{2}(\mathbf{k}, u)=\sum_{N=0}^{\infty} Z_{0}(N, \beta)\left\langle\sum_{j=1}^{N} \sum_{l=1}^{N} e^{i \mathbf{k}\left(\mathbf{r}_{j}-\mathbf{r}_{l}\right)} \Theta(l \neq j)\right\rangle u^{N} .
\end{gathered}
$$

For these generating functions, the summations and integrations can be performed analytically as for the isotropic case $[1,26]$. Using the notations $b_{i}=\exp \left\{-\beta \hbar w_{i}\right\}$ for $i=x, y, z$ and $\beta=1 / k_{B} T$, the generating functions are given by

$$
\begin{aligned}
\Xi(u)= & \exp \left\{\sum_{l=1}^{N} \frac{u^{l}\left(b_{x} b_{y} b_{z}\right)^{l / 2}}{\left(1-b_{x}^{l}\right)\left(1-b_{y}^{l}\right)\left(1-b_{z}^{l}\right)}\right\}, \quad(\mathrm{A} \\
G_{1}(\mathbf{k}, u)= & \Xi(u) \sum_{l=1}^{N} \frac{u^{l}\left(b_{x} b_{y} b_{z}\right)^{l / 2}}{\left(1-b_{x}^{l}\right)\left(1-b_{y}^{l}\right)\left(1-b_{z}^{l}\right)} \\
& \times \exp \left\{-\sum_{i=x, y, z} \frac{\hbar k_{i}^{2}}{4 m w_{i}} \frac{1+b_{i}^{l}}{1-b_{i}^{l}}\right\}, \\
G_{2}(\mathbf{k}, u)= & \Xi(u) \sum_{l=2}^{N} \frac{u^{l}\left(b_{x} b_{y} b_{z}\right)^{l / 2}}{\left(1-b_{x}^{l}\right)\left(1-b_{y}^{l}\right)\left(1-b_{z}^{l}\right)} \\
& \times \sum_{j=1}^{l-1}\left[\exp \left(-\sum_{i=x, y, z} \frac{\hbar k_{i}^{2}}{2 m w_{i}} P_{l, j}\left(b_{i}\right)\right)\right. \\
& \left.+\prod_{i=x, y, z} P_{l, j}^{-1}\left(b_{i}\right) \exp \left(-\frac{\hbar k_{i}^{2}}{2 m w_{i}} \frac{1}{P_{l, j}\left(b_{i}\right)}\right)\right],
\end{aligned}
$$

with $P_{l, j}(b)=\left(1-b^{j}\right)\left(1-b^{l-j}\right) /\left(1-b^{l}\right)$. Two schemes have been used to retrieve the expectation values from their generating function. The contour-integration scheme [38] was applied in [39], and yields

$$
Z_{0}(N, \beta)=\left.\frac{1}{N !} \frac{d^{N} \Xi(u)}{d u^{N}}\right|_{u=0}=\frac{1}{2 \pi} \int_{0}^{2 \pi} \frac{\Xi\left(u e^{i \theta}\right)}{u^{N}} e^{-i N \theta} d \theta,
$$

$$
\begin{aligned}
& \left\langle\sum_{j=1}^{N} e^{i \mathbf{k} \cdot \mathbf{r}_{j}}\right\rangle=\left.\frac{1}{Z_{0}(N, \beta)} \frac{1}{N !} \frac{d^{N} G_{1}(u)}{d u^{N}}\right|_{u=0} \\
& =\frac{\Xi(u) / u^{N}}{Z_{0}(N, \beta)} \frac{1}{2 \pi} \int_{0}^{2 \pi} \frac{G_{1}\left(u e^{i \theta}\right)}{\Xi(u)} e^{-i N \theta} d \theta, \\
& \left\langle\sum_{j=1}^{N} \sum_{l=1}^{N} e^{i \mathbf{k}\left(\mathbf{r}_{j}-\mathbf{r}_{l}\right)} \Theta(l \neq j)\right\rangle \\
& =\left.\frac{1}{Z_{0}(N, \beta)} \frac{1}{N !} \frac{d^{N} G_{2}(u)}{d u^{N}}\right|_{u=0} \\
& =\frac{\Xi(u) / u^{N}}{Z_{0}(N, \beta)} \frac{1}{2 \pi} \int_{0}^{2 \pi} \frac{G_{2}\left(u e^{i \theta}\right)}{\Xi(u)} e^{-i N \theta} d \theta .
\end{aligned}
$$

In principle, any value can be chosen for $u$. Fastest numerical convergence, however, is achieved for the steepest descent solution, when $u$ is chosen such that $N=$ $-\beta^{-2} \partial[\ln \Xi(u)] / \partial(\ln u)$. It would be incorrect to put the expectation value equal to the generating function itself, in which the steepest descent value $u=e^{\beta \mu}$ for the parameter $u$ in the free energy generating function, is substituted.

The second method is to collect the coefficient of $u^{N}$. This results in recursion relations between the expectation value for $N$ particles and the expectation values for smaller number of particles:

$$
\begin{aligned}
Z_{0}(N, \beta)= & \sum_{l=1}^{N} \frac{\left(b_{x} b_{y} b_{z}\right)^{l / 2}}{\left(1-b_{x}^{l}\right)\left(1-b_{y}^{l}\right)\left(1-b_{z}^{l}\right)} \\
& \times Z_{0}(N-l, \beta) \quad \text { with } Z_{0}(0, \beta)=1, \\
\left\langle\sum_{j=1}^{N} e^{i \mathbf{k} \cdot \mathbf{r}_{j}}\right\rangle= & \sum_{l=1}^{N} \frac{Z_{0}(N-l, \beta)\left(b_{x} b_{y} b_{z}\right)^{l / 2}}{Z_{0}(N, \beta)\left(1-b_{x}^{l}\right)\left(1-b_{y}^{l}\right)\left(1-b_{z}^{l}\right)} \\
& \left.\times \exp \left\{-\sum_{i=x, y, z} \frac{\hbar k_{i}^{2}}{4 m w_{i}} \frac{1+b_{i}^{l}}{1-b_{i}^{l}}\right\}, \quad \text { A2 }\right) \\
\left.\sum_{j=1}^{N} \sum_{l=1}^{N} e^{i \mathbf{k}\left(\mathbf{r}_{j}-\mathbf{r}_{l}\right)} \Theta(l \neq j)\right\rangle & \sum_{l=2}^{N} \frac{Z_{0}(N-l, \beta)\left(b_{x} b_{y} b_{z}\right)^{l / 2}}{Z_{0}(N, \beta)\left(1-b_{x}^{l}\right)\left(1-b_{y}^{l}\right)\left(1-b_{z}^{l}\right)} \\
& \times \sum_{j=1}^{l-1}\left[\exp \left(-\sum_{i=x, y, z} \frac{\hbar k_{i}^{2}}{2 m w_{i}} P_{l, j}\left(b_{i}\right)\right)\right. \\
& \left.+\prod_{i=x, y, z} P_{l, j}^{-1}\left(b_{i}\right) \exp \left(-\frac{\hbar k_{i}^{2}}{2 m w_{i}} \frac{1}{P_{l, j}\left(b_{i}\right)}\right)\right] .
\end{aligned}
$$


The numerical implementation of the recursion relations gives high accuracy at the cost of computing time. In practice, they become too time consuming for more than a few thousand particles, and expressions (A18)-(A20) have then to be computed. In summary, the expectation values in the path-integral formalism were calculated by introducing the generating functions (A12)-(A14) for these expectation values. By constructing the generating functions, one can analytically perform the sum over all permutations, necessary to incorporate the quantum statistics. The expectation value can then be extracted from the generating function using the inversion formulas (A18)-(A20) or (A21)-(A23).
[1] F. Brosens, J. T. Devreese, and L. F. Lemmens, Phys. Rev. E 55, 6795 (1997).

[2] J. Tempere, F. Brosens, L. F. Lemmens, and J. T. Devreese, Solid State Commun. 107, 51 (1998).

[3] M. H. Anderson, J. R. Ensher, M. R. Matthews, C. E. Wieman, and E. A. Cornell, Science 269, 198 (1995).

[4] K. B. Davis, M.-O. Mewes, M. R. Andrews, N. J. Van Druten, D. S. Durfee, D. M. Kurn, and W. Ketterle, Phys. Rev. Lett. 75, 3969 (1995).

[5] C. C. Bradley, C. A. Sackett, J. J. Tollett, and R. G. Hulet, Phys. Rev. Lett. 75, 1687 (1995).

[6] F. Dalfovo and S. Stringari, Phys. Rev. A 53, 2477 (1996).

[7] M. Houbiers and H. T. C. Stoof, Phys. Rev. A 54, 5055 (1996).

[8] C. A. Sackett, H. T. C. Stoof, and R. G. Hulet, Phys. Rev. Lett. 80, 2031 (1998).

[9] S. Giorgini, L. P. Pitaevskii, and S. Stringari, Phys. Rev. A 54, R4633 (1996).

[10] S. Giorgini, L. P. Pitaevskii, and S. Stringari, Phys. Rev. Lett. 78, 3987 (1997).

[11] D. A. W. Hutchinson, E. Zaremba, and A. Griffin, Phys. Rev. Lett. 78, 1842 (1997).

[12] A. Minguzzi, S. Conti, and M. P. Tosi, J. Phys.: Condens. Matter 9, L33 (1997).

[13] H. Shi and W.-M. Zheng, Phys. Rev. A 56, 1046 (1997).

[14] G. Baym and C. Pethick, Phys. Rev. Lett. 76, 6 (1996).

[15] N. P. Proukakis and K. Burnett, J. Res. Natl. Inst. Stand. Technol. 101, 457 (1996).

[16] M. Bijlsma and H. T. C. Stoof, Phys. Rev. A 55, 498 (1997).

[17] A. Griffin, Phys. Rev. B 53, 9341 (1996).

[18] R. P. Feynman, Phys. Rev. 97, 660 (1955).

[19] D. S. Hall, M. R. Matthews, C. E. Wieman, and E. A. Cornell, Phys. Rev. Lett. 81, 1543 (1998).

[20] E. R. I. Abraham, W. I. McAlexander, H. T. C. Stoof, and R. G. Hulet, Phys. Rev. A 53, 3092 (1996).

[21] E. R. I. Abraham, W. I. McAlexander, J. M. Gerton, R. G. Hulet, R. Coté, and A. Dalgarno, Phys. Rev. A 55, 3299 (1997).

[22] E. R. I. Abraham, W. I. McAlexander, C. A. Sackett, and R. G. Hulet, Phys. Rev. Lett. 74, 1315 (1995).

[23] I. M. Torrens, Interatomic Potentials (Academic Press, New York, 1972); L. P. Pitaevskii, in Proceedings of the International School of Physics "Enrico Fermi," Course CXL,
Varenna, 1998, edited by M. Inguscio, S. Stringari, and C. Wieman (IOS Press, Amsterdam, 1999).

[24] B. Simon, Functional Integration and Quantum Physics (Academic Press, New York, 1979).

[25] R. P. Feynman, Statistical Mechanics: A Set of Lectures (Benjamin, Reading, MA, 1972).

[26] F. Brosens, J. T. Devreese, and L. F. Lemmens, Phys. Rev. E 55, 227 (1997).

[27] For the scattering lengths of the alkali gasses we used the following sources: E. R. I. Abraham et al., Phys. Rev. Lett. 74, 1315 (1995) for lithium; K. B. Davis et al., ibid. 74, 5202 (1995) and A. J. Moerdijk et al., ibid. 73, 518 (1994) for sodium; N. R. Newbury et al., Phys. Rev. A 51, 2680 (1995) for rubidium-87; Gardner et al., Phys. Rev. Lett. 74, 3764 (1995) for rubidium-85; C. R. Monroe et al., ibid. 70, 414 (1993) and M. Arndt et al., ibid. 79, 625 (1997) for cesium.

[28] Typical confinement frequencies were taken from C. C. Bradley et al., Phys. Rev. Lett. 78, 985 (1997) for lithium; K. B. Davis et al., ibid. 74, 5202 (1995) for sodium; D. S. Jin et al., ibid. 78, 764 (1997) for rubidium-87; Gardner et al., ibid. 74, 3764 (1995) for rubidium-85; M. Arndt et al., ibid. 79, 625 (1997) for cesium.

[29] L. Salasnic, Mod. Phys. Lett. B 11, 1249 (1997).

[30] C. C. Bradley, C. A. Sackett, and R. G. Hulet, Phys. Rev. Lett. 78, 985 (1997).

[31] R. Côté, A. Dalgarno, and M. J. Jamieson, Phys. Rev. A 50, 399 (1994).

[32] W. Krauth, Phys. Rev. Lett. 77, 3695 (1996).

[33] P. Gruter, D. Ceperley, and F. Laloë, Phys. Rev. Lett. 79, 3549 (1997).

[34] J. Williams, R. Walser, C. Wieman, J. Cooper, and M. Holland, Phys. Rev. A 57, 2030 (1998).

[35] C. A. Sackett, C. C. Bradley, M. Welling, and R. G. Hulet, Appl. Phys. B 65, 433 (1997).

[36] E. A. Burt, R. W. Ghrist, C. J. Myatt, M. J. Holland, E. A. Cornell, and C. E. Wieman, Phys. Rev. Lett. 79, 337 (1997).

[37] Y. Kagan, B. V. Shustunov, and G. V. Shlyapnikov, Pis'ma Zh. Éksp. Teor. Fiz. 42, 169 (1985) [JETP Lett. 42, 209 (1985)].

[38] R. Kubo, Statistical Mechanics (North-Holland, Amsterdam, 1974).

[39] L. F. Lemmens, F. Brosens, and J. T. Devreese, Solid State Commun. 109, 615 (1999). 